\title{
Complete sequencing of Novosphingobium sp. PP1Y reveals a biotechnologically meaningful metabolic pattern
}

\author{
Valeria D'Argenio ${ }^{1,2 \dagger}$, Eugenio Notomista ${ }^{3+}$, Mauro Petrillo ${ }^{1,2 \dagger}$, Piergiuseppe Cantiello ${ }^{1}$, Valeria Cafaro ${ }^{3}$, Viviana Izzo ${ }^{4}$, \\ Barbara Naso ${ }^{1,2}$, Luca Cozzuto ${ }^{1}$, Lorenzo Durante ${ }^{3}$, Luca Troncone ${ }^{3}$, Giovanni Paolella ${ }^{1,2}$, Francesco Salvatore ${ }^{1,5^{*}}$ \\ and Alberto Di Donato ${ }^{3}$
}

\begin{abstract}
Background: Novosphingobium sp. strain PP1Y is a marine a-proteobacterium adapted to grow at the water/fuel oil interface. It exploits the aromatic fraction of fuel oils as a carbon and energy source. PP1Y is able to grow on a wide range of mono-, poly- and heterocyclic aromatic hydrocarbons. Here, we report the complete functional annotation of the whole Novosphingobium genome.

Results: PP1Y genome analysis and its comparison with other Sphingomonadal genomes has yielded novel insights into the molecular basis of PP1Y's phenotypic traits, such as its peculiar ability to encapsulate and degrade the aromatic fraction of fuel oils. In particular, we have identified and dissected several highly specialized metabolic pathways involved in: (i) aromatic hydrocarbon degradation; (ii) resistance to toxic compounds; and (iii) the quorum sensing mechanism.

Conclusions: In summary, the unraveling of the entire PP1Y genome sequence has provided important insight into PP1Y metabolism and, most importantly, has opened new perspectives about the possibility of its manipulation for bioremediation purposes.
\end{abstract}

Keywords: De novo sequencing, Novosphingobium sp. PP1Y, Sphingomonads, Next generation sequencing, Aromatic pollutant compounds/bioremediation

\section{Background}

Aromatic compounds are among the most widespread dangerous pollutants [1]. Petroleum and its derivatives are the main sources of aromatic molecules released into the environment. The aromatic hydrocarbon content of petroleum can range from about $20 \%$ to more than $40 \%$ [2-4], whereas the aromatic hydrocarbon content of gasoline and diesel oil is about $30 \%$ and $25 \%$, respectively $[5,6]$.

Novosphingobium sp. strain PP1Y is a recently isolated marine $\alpha$-proteobacterium that is able to grow on a surprisingly wide spectrum of pure mono-, poly- and heterocyclic aromatic hydrocarbons and on complex mixtures of aromatic hydrocarbons dissolved in paraffin

\footnotetext{
* Correspondence: salvator@unina.it

${ }^{\dagger}$ Equal contributors

${ }^{1}$ CEINGE-Biotecnologie Avanzate, Napoli, Italy

${ }^{5}$ IRCCS-Fondazione SDN, Naples, Italy

Full list of author information is available at the end of the article
}

oil phases including gasoline and especially diesel-oil which is an optimal growth substrate. Moreover, PP1Y can emulsify diesel-oil by producing small $(<1 \mathrm{~mm})$ regular biofilm-covered oil drops that have been described as spherical colonies harbouring a reservoir of growth substrates [7].

Strain PP1Y belongs to the Sphingomonadaceae family, which is characterized by the presence of glycosphingolipids in the outer membrane, instead of the more common lipopolysaccharides. This peculiarity renders the surface of their cells more hydrophobic than those of the other Gram-negative strains and, has probably contributed to the development of the ability to degrade mono- and polycyclic aromatic hydrocarbons (PAHs). Moreover, many Sphingomonadales harbour several (up to six) large conjugative plasmids, ranging in length from less than $50 \mathrm{kbp}$ to more than $500 \mathrm{kbp}$ [8]. Thanks to these 
megaplasmids, several Sphingomonadales have "collected" genes for the degradation of xenobiotics and continuously exchange them with other bacterial strains [9-11]. Interesting examples are Novosphingobium aromaticivorans F199, which uses alkyl-benzenes as the sole carbon and energy source [12], Novosphingobium pentaromativorans US6-1, which degrades PAHs with 3-5 aromatic rings [13], Novosphingobium sp. TYA-1, which simultaneously degrades bisphenol A and 4-alkylphenols [14] Sphingomonas paucimobilis EPA505, which degrades several polycyclic compounds [15], Sphingomonas wittichii RW1, which can grow using dibenzofuran and dibenzo- $p$-dioxin [16], Sphingomonas sp. TTNP3 which uses alkylphenolic compounds as a source of carbon and energy [17] and Sphingobium chlorophenolicum L-1 which degrades pentachlorophenol [11].

Here, we report the analysis of the genome of Novosphingobium sp. strain PP1Y and its comparison with the genomes of $N$. aromaticivorans F199 (genome accession number NC_007794.1) [18] and S. wittichii RW1 [19], the closest genomes in terms of nucleotide sequence. This comparison has yielded insights into PP1Y and its ability to encapsulate and degrade the aromatic fraction of fuel oils.

\section{Results and discussion}

\section{Complete genome features and chromosomal} architecture

PP1Y genome sequence assembly produced four replicons classified according to their size, as we previously reported [20] (Figure 1A-D). Because the coverage of "small" plasmid ( $\mathrm{Spl}$ ) sequences was, on average, about twice that of the other replicons, it is expected that $\mathrm{Spl}$ is present as a two-copy object within each bacterial cell. At present, very few complete sequences of bacterial chromosomes and plasmids are available for organisms of the genus Novosphingobium (see Table 1). These sequences have a similar $\mathrm{G}+\mathrm{C}$ content (about 60\%), but PP1Y appears to have the largest and most complex genomic organization of the genus.

Various predictive and comparative bioinformatics tools supported by biological databases were used to annotate putative open reading frames (ORFs) and other functional elements [21-27]. As in other bacteria, most of the genome sequence is predicted to be coding and a substantial fraction of predicted ORFs (12-22\%, depending on the replicon) appear to have TTG or GTG as the starting codon. Most of them $(73 \%$ of the 4,709 coding sequences predicted in the four replicons taken together) and all rRNA and tRNA genes are located on the Chr molecule. The same applies to other RNA elements; the only exceptions being three RNAs predicted on Lpl (see Table 2, "Other RNA elements" section and Additional file 1: Table S1).

\section{Evaluation of the putative DNA replication origins}

DNA replication was investigated by searching for the putative genome replication origins using a bioinformatic tool. This tool, Orifinder [28], locates predicted bacterial replication origins within each DNA sequence by taking into account base composition asymmetry, distribution of DNA-A boxes and the presence of genes frequently located close to the bacterial replication start (Additional file 1: Table S1). This tool revealed a putative Type-III replication origin on $\mathrm{Chr}$, around base 1 , where there is a region of base composition asymmetry containing three DNA-A-boxes, close to the hemE gene (as in the $N$. aromaticivorans DSM 12444 genome) and to a DNA-A gene. Differently, on $\mathrm{Mpl}, \mathrm{Lpl}$ and Spl replicons, Orifinder failed to locate an acceptable putative replication origin, suggesting that other mechanisms may be involved in DNA replications origins. Interestingly, a typical plasmid replication parA/parB/parS cluster was found on each of these replicons, and $\mathrm{Mpl}$ and $\mathrm{Lpl}$ contain also a predicted plasmid replication repA gene close to the $\mathrm{parA} / \mathrm{parB} /$ parS cluster, but in a different orientation to those predicted on the $N$. aromaticivorans DSM 12444 pNL1 and pNL2 plasmids.

On the Spl plasmid. a complete protein killer gene system is also found, namely, an operon containing two genes that force the host bacteria to retain the plasmid [29].

\section{Protein genes identified and their significance}

The gene products encoded by the 4,709 ORFs were characterized by searching for sequence similarity with known bacterial proteins contained in various collections (Figure 2A). About 94\% of the ORFs matched at least one protein stored in the Uniref50 or KEGG GENES databases, although the fraction of matched sequences varies and is significantly lower for $\mathrm{Lpl}$ and Spl (about $80 \%$ ). It is noteworthy that about $20 \%$ of the ORFs matched proteins annotated as "hypothetical", "putative" or "uncharacterized", and are thus classified as coding for "conserved hypothetical proteins". When the same search was done against protein sequences stored in the COG database, the fraction of identified gene products was lower. In fact, most of the ORFs coding for "conserved hypothetical proteins" did not show any similarity. About $6 \%$ of the ORFs did not match any sequence stored in the three databases and are thus classified as coding for "hypothetical proteins".

An all-against-all comparison of the protein sequences encoded within each replicon was done using BLAST [30] under very stringent conditions (see Table 3, PP1Y-PP1Y section, and Additional file 1: Figure S1A) to look for inter-duplicated genes. About 20\% of ORFs from Mpl have a counterpart within the main chromosome (Chr), thereby indicating a partial genome duplication. The 


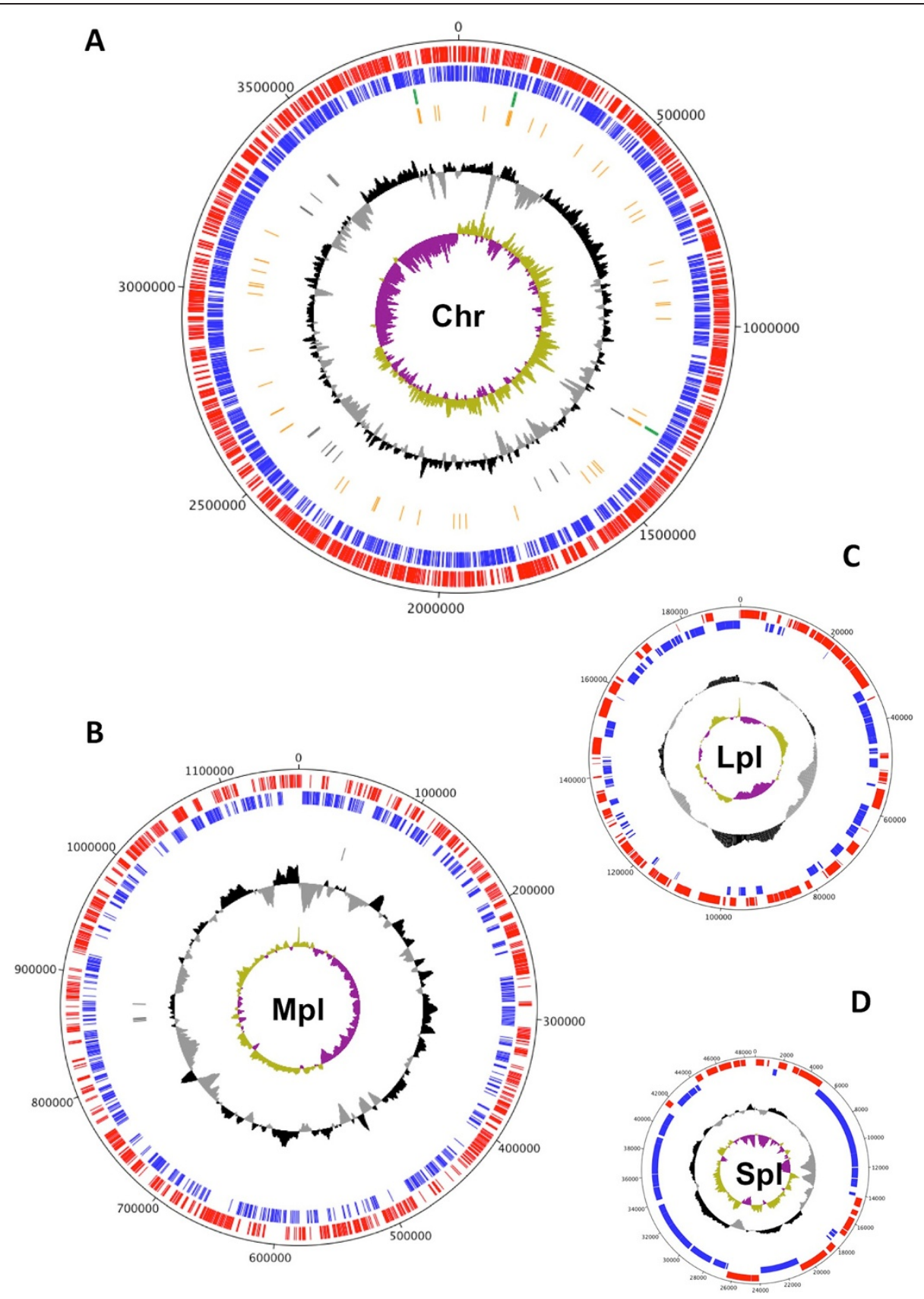

Figure 1 Circular maps and genetic features of PP1Y replicons. The principal genomic features of PP1Y Chr (A) and its plasmids (B, C and D) are shown. For each of them, we report, from outside to the center, genes on the forward and on reverse strands (red and blue), GC content (black) and GC skew (green and violet).

whole complement of protein-coding genes was also compared to the one encoded within other complete genomes and plasmids from bacteria of the Sphingomonadaceae family (Table 3 and Additional file 1: Figure S1B-D). A number of ORFs ranging between 1,500 and 1,700, i.e. 45-50\% of those encoded within Chr in PP1Y, have a counterpart in the main chromosome of the closest analysed bacterial species, the most similar gene set being that from $N$. aromaticivorans, putatively from the same genus. There is no clear evidence that the three smaller replicons are functionally equivalent to other known plasmids in terms of protein coding genes: many protein genes predicted within $\mathrm{Mpl}$ appear to have counterparts in $N$. aromaticivorans, although only $20 \%$ of Lpl ORFs have a counterpart in the pNL1 plasmid (Accession: NC_009426.1), while others are in the pNL2 plasmid (Accession: NC_009427.1) and some are scattered along the main chromosome. Half the Spl-encoded 
Table 1 Principal features and comparison of the genomic sequences available for bacteria of the genus Novosphingobium

\begin{tabular}{|c|c|c|c|c|c|c|c|c|c|c|c|}
\hline Species & Sequence complete & Sequence available & Size (Mb) & Chrs & Plasmids & Scaffolds & Contigs & CDS & 16SrRNA & Identities & Gaps \\
\hline Novosphingobium sp. PP1Y & yes & yes & 5,073 & 1 & 3 & - & - & yes & yes & $1,477 / 1,477$ & $0 / 1,477$ \\
\hline Novosphingobium sp. AP12 & no & yes & 5,611 & - & - & - & 187 & yes & yes & $1,437 / 1,480$ & $6 / 1,480$ \\
\hline Novosphingobium sp. Rr 2-17 & no & yes & 4,539 & - & - & - & 166 & yes & yes & $1,424 / 1,480$ & $6 / 1,480$ \\
\hline Novosphingobium pentaromativorans US6-1 ${ }^{\text {a }}$ & no & yes & 5,096 & - & 2 & - & 123 & yes & yes & $1,476 / 1,477$ & $0 / 1,477$ \\
\hline Sphingobium chlorophenolicum L-1 & yes & yes & 4,449 & 2 & 1 & - & - & yes & yes & $1,360 / 1,480$ & $7 / 1,480$ \\
\hline Sphingobium indicum B90A & no & yes & 4,082 & - & - & - & 149 & yes & yes & $1,373 / 1,480$ & $7 / 1,480$ \\
\hline Sphingomonas echinoides ATCC 14820 & no & yes & 4,200 & - & - & 6 & 64 & yes & yes & $1,372 / 1,488$ & $26 / 1,488$ \\
\hline Sphingomonas wittichii DP58 & no & yes & 5,628 & - & - & - & 739 & no & yes & $1,372 / 1,484$ & $16 / 1,484$ \\
\hline Sphingomonas wittichii RW1 & yes & yes & 5,382 & 1 & 2 & - & - & yes & yes & $1,373 / 1,484$ & $16 / 1,484$ \\
\hline Novosphingobium aromaticivorans DSM 12444 & yes & yes & 3,561 & 1 & 2 & - & - & yes & yes & $1,400 / 1,480$ & $10 / 1,480$ \\
\hline Novosphingobium nitrogenifigens DSM 19370 B057DRAFT & no & yes & 4,148 & - & - & - & 48 & yes & yes & $1,399 / 1,478$ & $29 / 1,478$ \\
\hline Novosphingobium nitrogenifigens DSM 19370 & no & yes & 4,140 & - & - & - & 109 & yes & yes & $1,400 / 1,479$ & $31 / 1,479$ \\
\hline
\end{tabular}

${ }^{a}$ no plasmids plA1 and pIA2 in the size count; ${ }^{\mathrm{b}} \mathrm{chr} 1+\mathrm{chr} 2$ in the size count.

PP1Y strain is in boldface type. 
Table 2 Genome sequencing statistics of the entire PP1Y genome

\begin{tabular}{|c|c|c|c|c|}
\hline & Chr & Mpl & Lpl & Spl \\
\hline \multicolumn{5}{|l|}{ Genome } \\
\hline Size, bp & $3,911,486$ & $1,161,602$ & 192,103 & 48,714 \\
\hline $\mathbf{G}+\mathbf{C}$ content, $\%$ & 63.7 & 62.3 & 60.7 & 60.1 \\
\hline Copies for each bacterial cell & 1 & 1 & 1 & 2 \\
\hline \multicolumn{5}{|l|}{ ORFS } \\
\hline Number & 3,481 & 975 & 199 & 54 \\
\hline Minimum length, bp & 100 & 100 & 100 & 100 \\
\hline Average length, bp & 990 & 1,059 & 788 & 776 \\
\hline Maximum length, bp & 7,749 & 3,597 & 4,479 & 5,748 \\
\hline ATG initiation codons, $\%$ & 86.8 & 88.4 & 76.4 & 81.5 \\
\hline GTG initiation codons, $\%$ & 8.8 & 7.5 & 12.0 & 7.4 \\
\hline TTG initiation codons, $\%$ & 4.4 & 4.1 & 11.6 & 11.1 \\
\hline$\%$ of coding sequence & 88.1 & 88.9 & 81.6 & 86.0 \\
\hline \multicolumn{5}{|l|}{ Repetitive elements } \\
\hline Prophage elements & 5 & 1 & 1 & 1 \\
\hline IS elements & 3 & 5 & 7 & 0 \\
\hline \multicolumn{5}{|l|}{ RNAs } \\
\hline rRNA (16S-23S-5S) & 3 & 0 & 0 & 0 \\
\hline tRNAs & 58 & 0 & 0 & 0 \\
\hline Other RNA elements* & 15 & 0 & 3 & 0 \\
\hline Cobalamin riboswitches & 5 & 0 & 1 & 0 \\
\hline suhB & 3 & 0 & 0 & 0 \\
\hline SRP_bact & 1 & 0 & 0 & 0 \\
\hline Rnase P clA & 1 & 0 & 0 & 0 \\
\hline tmRNA & 1 & 0 & 0 & 0 \\
\hline TPP riboswitch & 1 & 0 & 0 & 0 \\
\hline ctRNA p42d & 1 & 0 & 0 & 0 \\
\hline Glycine riboswitch & 1 & 0 & 0 & 0 \\
\hline LR-PK1 & 1 & 0 & 0 & 0 \\
\hline Groupll catalytic introns & 0 & 0 & 1 & 0 \\
\hline ROSE & 0 & 0 & 1 & 0 \\
\hline
\end{tabular}

*Predicted RNA elements, besides tRNAs and rRNAs.

proteins are encoded by the main chromosome in $N$. aromaticivorans. Sphingobium japonicum [31] and PP1Y share elements of comparable size, although the latter has an additional smaller chromosome. The two species have a $45 \%$ similarity within the main chromosome in terms of protein-encoding content, but diverge more extensively in the plasmids. A plasmid from S. japonicum UT26 pLB1 [32], which is involved in gamma-hexachlorocyclohexane degradation, is somewhat similar to $\mathrm{Spl}$ (data not shown).

To assign a putative biological function to protein-coding genes, they were classified, when possible, into COG functional categories based on the result of a BLAST search against COG genes. The predicted protein sequences were also analyzed with the KEGG Automatic Annotation Server KAAS, which assigns a functional annotation to genes following a BLAST alignment against the manually curated KEGG genes database [33] (Additional file 1: Figure S2 A-B). Overall, the Chr sequence of PP1Y contains practically all the core metabolism genes; notably, a number of predicted transporters and transcription factors are present in $\mathrm{Mpl}$ (Figure 2B-C).

\section{Characterization of the PP1Y genes involved in aromatic hydrocarbon degradation}

The degradation of aromatic hydrocarbons requires activation of the aromatic ring. This generally occurs by dihydroxylation of the aromatic ring catalyzed by pairs of monooxygenases or dioxygenases/dehydrogenases that constitute the upper pathways. Ring activation is followed by ring cleavage catalyzed by specialized dioxygenases (intra- and extradiol dioxygenases) that start the lower pathways. In the case of methylated aromatic compounds, the initial step can be a monooxygenation reaction of a methyl group followed by oxidation to carboxylate. These reactions can be catalyzed by soluble dioxygenases or by membrane monoxygenases related to xylene monooxygenase XylM. The arylcarboxylate eventually undergoes ring dihydroxylation and cleavage [34]. Analysis of the PP1Y genome revealed at least 81 ORFs (Table 4) that potentially code for the enzymes of both the upper (ring activation) and lower (ring cleavage) pathways.

No soluble multicomponent monooxygenase that resembled the well characterized methane monooxygenases and toluene/o-xylene monooxygenase [35] was found in the present study. Thirty-eight ORFs, which were predicted to code for 34 different multicomponent aromatic hydroxylating dioxygenases [36], were identified - a number clearly higher than in the closely related strains $N$. aromaticivorans F199 and $N$. pentaromativorans US6-1 (27 and 18 dioxygenases, respectively) (Figure 3). PPIY has a close counterpart of each F199 dioxygenase: three of these are present in double copy with a $100 \%$ identity, which is indicative of a very recent duplication event; and four others have a 90-95\% identity, which suggests a less recent duplication event followed by divergence. All duplicated ORFs are closely related to seven ORFs coding for hydroxylating dioxygenases found on plasmid pNL1 from strain F199. Indeed, replicon A of strain PP1Y contains two copies of a region of plasmid pNL1 probably derived by multiple fusion/duplication events (Additional file 1: Figure S3A). Six PP1Y oxygenases from the megaplasmids (Mpl6792, Mpl2166, Mpl5621, Mpl5540, Mpl5477, Mpl5466) do not have homologues in strains F199 and US6-1 but are closely related to predicted oxygenases from strain RW1 (Additional file 1: Figure S4A and B), suggesting that strain PP1Y combined the 

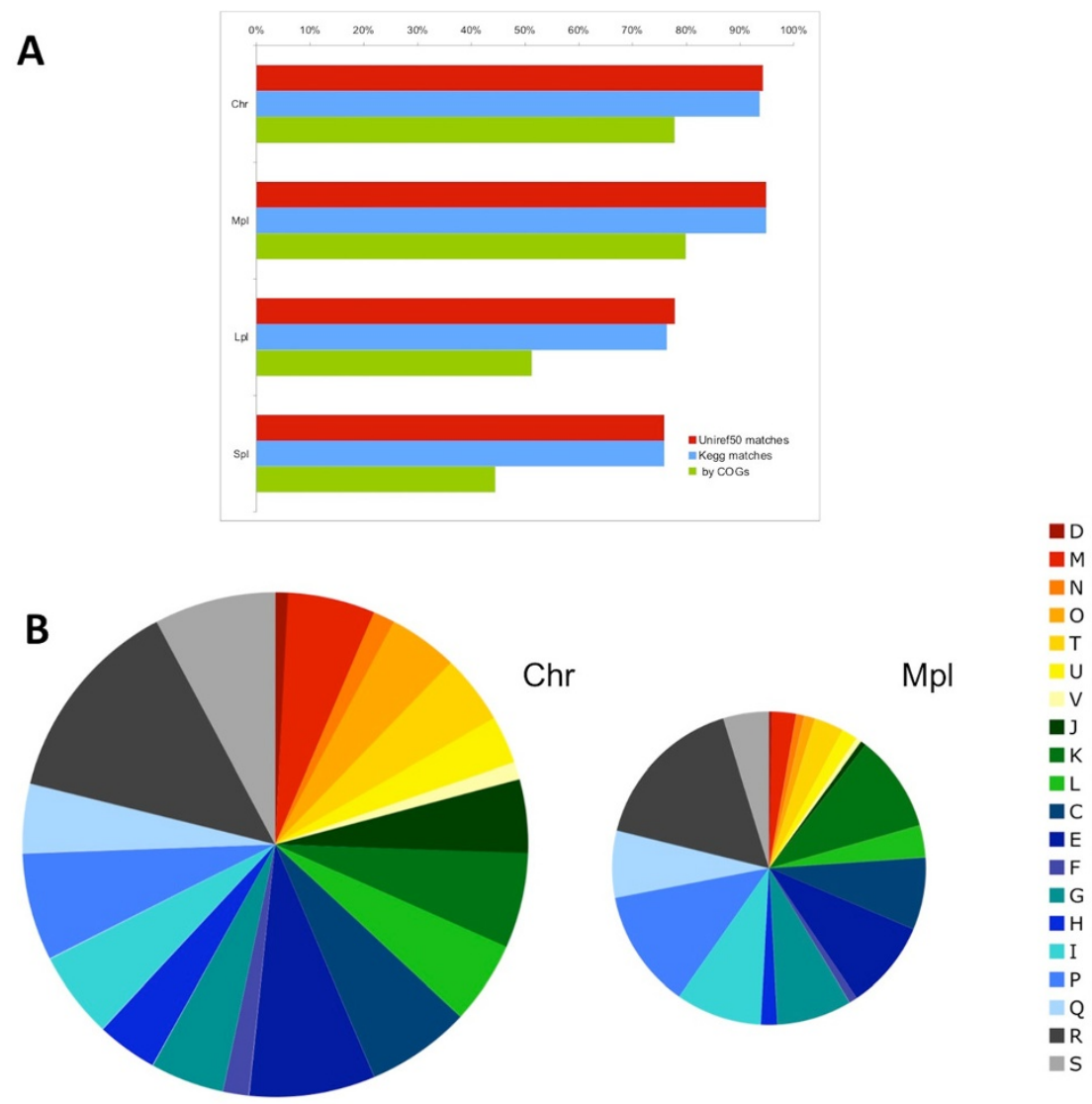

C

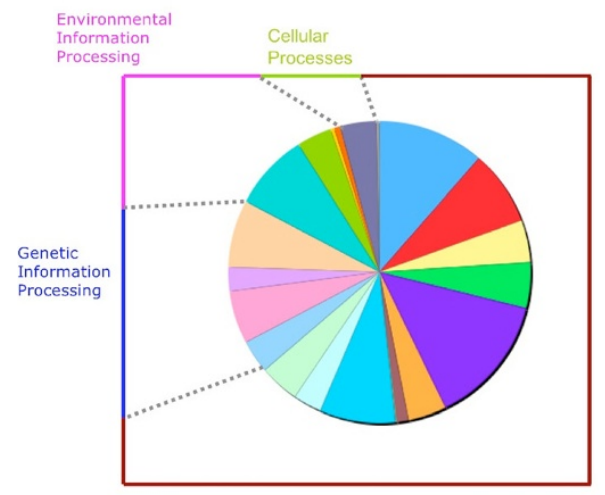

Metabolism

$\mathrm{Chr}$

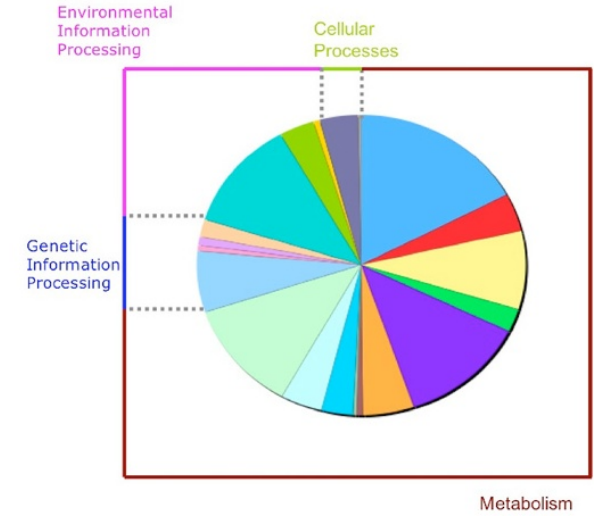

Figure 2 Open reading frame (ORF) annotation. Fraction of ORFs that resemble another protein based on BLAST matches with the Uniref50 and KEGG databases and that have a predicted function according to COG categories (A). The distribution of Chr and Mpl genes is reported with respect to COG functional categories (B) and according to Kegg pathways (C). D, Cell cycle control, cell division, chromosome partitioning. M, Cell wall/membrane/envelope biogenesis; N, Cell motility; O, Posttranslational modification, protein turnover, chaperones; T: Signal transduction mechanisms; U: Intracellular trafficking, secretion, and vesicular transport; $\mathrm{V}$, Defense mechanisms; J: Translation, ribosomal structure and biogenesis; K: Transcription; L: Replication, recombination and repair; C, Energy production and conversion; E, Amino acid transport and metabolism; F, Nucleotide transport and metabolism; G, Carbohydrate transport and metabolism; H, Coenzyme transport and metabolism; I, Lipid transport and metabolism; P, Inorganic ion transport and metabolism; Q, Secondary metabolite biosynthesis, transport and catabolism; R, General function prediction only; S. Function unknown. 
Table 3 Predicted protein genes comparison

\begin{tabular}{|c|c|c|c|c|c|c|}
\hline \multirow[b]{2}{*}{ Genome } & \multirow[b]{2}{*}{ Replicon } & \multirow[t]{2}{*}{ Total ORFs } & \multicolumn{4}{|c|}{ ORFs in PP1Y replicons } \\
\hline & & & Chr & Mpl & Lpl & Spl \\
\hline \multirow{4}{*}{ PP1Y } & Chr & 3,481 & 3,481 & 203 & 30 & 4 \\
\hline & $\mathrm{Mpl}$ & 975 & 240 & 975 & 10 & - \\
\hline & Lpl & 199 & 43 & 10 & 199 & - \\
\hline & Spl & 54 & 5 & - & - & 54 \\
\hline \multirow{3}{*}{ N.aro } & Chr & 3,324 & 1,741 & 296 & 43 & 22 \\
\hline & pNL1 & 182 & 203 & 32 & 8 & - \\
\hline & pNL2 & 431 & 204 & 232 & 5 & - \\
\hline E.lit & Chr & 3,012 & 1,513 & - & - & - \\
\hline S.ala & Chr & 3,166 & 1,536 & - & - & - \\
\hline S.wit & Chr & 4,851 & 1,543 & - & - & - \\
\hline Z.mob1 & Chr & 1,801 & 885 & - & - & - \\
\hline Z.mobZ & Chr & 1,729 & 865 & - & - & - \\
\hline \multirow{5}{*}{ S.jap } & Chr1 & 3,530 & 1,573 & - & - & - \\
\hline & Chr2 & 590 & 234 & 147 & 0 & - \\
\hline & $\mathrm{pCHQ} 1$ & 225 & 102 & 11 & 23 & - \\
\hline & pUT1 & 45 & 8 & - & - & - \\
\hline & pUT2 & 9 & - & - & - & - \\
\hline
\end{tabular}

N. aro = Novosphingobium aromaticivorans DSM 12444; E. lit = Erythrobacter litoralis; S. ala = Sphingopyxis alaskenis; $\mathrm{S}$. wit = Sphingomonas wittichii; Z. mob $=$ Zymomonas mobilis $11163 ;$ Z. $\mathrm{mobZ}=$ Zymomonasmobilis ZM4; $S$. jap $=$ Sphingonium japonicum.

The protein-coding genes identified in PP1Y are compared with those of several genomes and plasmids.

dioxygenase pools of strains F199 and RW1 and later expanded the pool by duplication events. This strategy enabled PP1Y to expand the pathway for the degradation of naphthalene and methylnaphthalenes, and to degrade larger PAHs. The predicted pathway is shown in Additional file 1: Figure S3B.

Two potential membrane monooxygenases are predicted in PP1Y; they show a $96 \%$ identity with each other and a $71-75 \%$ identity with the sole membrane monooxygenase found in strain F199, which suggests another recent event of gene duplication. The two PP1Y monooxygenases (Additional file 1: Figure S5) mainly differ in the substrate-binding region, possibly to allow different substrate specificity. No membrane monooxygenase is present in the genomes of Sphingomonas sp. MM-1, Sphingobium japonicum UT26, Sphingobium chlorophenolicum L-1, Sphingobium sp. SYK-6, Sphingobium wittichii RW1 or Novosphingobium pentaromativorans US6-1. This suggests that, also in this case, the PP1Y enzymatic repertoire was expanded by horizontal gene transfer and duplication events.

Ten potential ORFs code for seven homomultimeric extradiol ring cleavage dioxygenases (RCDs) [37], related to Pseudomonas putida MT2 catechol 2,3-dioxygenase (Additional file 1: Figure S6). ORF AT15671/AT31616 codes for a putative classic catechol 2,3-dioxygenase; homology modeling and substrate docking have an active site pocket slightly larger than the family benchmark P. putida MT2 catechol 2,3-dioxygenase (not shown). AT15599/AT31688 code for a putative dihydroxynaphthalene dioxygenase, and Mpl3065 for a putative 2,3-dihydroxybiphenyl-1,2-dioxygenase. AT32663 is a divergent member of the extradiol dioxygenase family and no closely related sequence is present in the protein databases.

Homology models of these three RCDs are shown in Additional file 1: Figure S7. The four RCDs were cloned, expressed in Escherichia coli and their cleavage activity was assayed on 3-methylcatechol, 2,3-dihydroxybiphenyl (2,3-DHBP), and 4-hydroxyoestradiol (4-OHE). The latter was used as analogue of dihydroxy PAHs because these compounds are unstable, difficult to synthesize and not commercially available. The protein coded in AT15599/ AT31688 is a very versatile enzyme, able to cleave substrates with 1 to 4 rings (Table 5). Enzyme AT32663 is only active on polycyclic substrates, while Mpl3065 is active only on 2,3-DHBP, as predicted. Finally, AT15671/AT31616 is very active on monocyclic catechols, even though its substrate specificity is wider than that of $P$. putida MT2 catechol 2,3-dioxygenase. Taken together, these four enzymes are able to cleave all classes of 3- and/or 4-substituted catechols in complex mixtures. The other three PP1Y RCDs, AT33026, Mpl10251 and Mpl4329/Mpl4634, are poorly characterized. Preliminary modelling studies suggest that they are dioxygenases specialized in cleaving catechols bearing substituents at positions 3,5 and/or 4,5 and/or 3,6. Therefore, these dioxygenases have a substrate specificity complementary to the four described above.

The Neighbor-Joining tree of RCDs (Additional file 1: Figure S6) shows a great heterogeneity among sphingomonads both in the number of potential RCDs (from 1, in the case of strain L-1, to 8 in the case of strain RW1) and in the distribution of the proteins among the RCD subfamilies. Only strains F199 and PP1Y have at least one representative for each subfamily. This particular set of RCDs could allow strain PP1Y to metabolize complex mixtures of catechols deriving from the simultaneous oxidation of several mono- and polycyclic-aromatic hydrocarbons (Additional file 1: Figure S8), which are the preferred substrates for growing this strain.

Besides the seven homomultimeric estradiol RCDs, the PP1Y genome contains also four potential ORFs for heterodimeric extradiol RCDs that are able to cleave catechol rings bearing substituents with carboxylate groups like protocatechuate (see also Additional file 2: Supplementary Results and Discussion). The genome of strain PP1Y contains several other ORFs coding for hypothetical mono- and dioxygenases whose involvement in the degradation of xenobiotics is less clear. Among these, CDS AT10830 is particularly interesting as it codes for a 2-oxoglutarate-dependent oxygenase. These oxygenases 
Table 4 Number of ORFs coding for potential upper (ring activation) and lower (ring cleavage) pathway enzymes in strains PP1Y and F199

\begin{tabular}{lcc}
\hline & Novosphingobium sp. PP1Y & Novosphingobium aromaticivorans F199 \\
\hline Ring hydroxylating dioxygenases & $38^{\text {a }}$ & 27 \\
Membrane-bound monooxygenases & 2 & 1 \\
Molybdopteryn-dependent monooxygenases & 5 & 3 \\
Flavine monooxygenases & 19 & 1 \\
2-oxoglutarate-dependent oxygenases (taurine-dioxygenase like) & 2 & 0 \\
2-oxoglutarate-dependent oxygenases & $10^{\text {a }}$ & 1 \\
Extradiol ring-cleavage dioxygenases & 4 & 6 \\
Protocatechuate 4,5 dioxygenases (estradiol ring-cleavage) & & 3
\end{tabular}

n.d., not determined.

${ }^{\mathrm{a}}$ Four ORFs coding for ring hydroxylating dioxygenases and 3 ORFs coding for extradiol ring-cleavage dioxygenases are duplicated, therefore, the genome of PP1Y codes for 34 potential ring hydroxylating dioxygenases and 7 potential extradiol ring-cleavage dioxygenases.

cleave different substrates, namely alkyl-sulphonates and fenoxy-acids, by catalyzing monooxygenation reactions of $\mathrm{CH}$ bonds adjacent to good leaving groups. Interestingly, no sphingomonad contains a homologous enzyme. Moreover, AT10830 is a member of a group of adjacent ORFs coding for: (i) a hydroxylating dioxygenase (AT10866) that is only distantly related to RW1 and F199 dioxygenases (Additional file 1: Figure S4A); (ii) a heterodimeric extradiol ring cleavage dioxygenase related to 3,4-dihydroxybenzoate dioxygenases; and (iii) a hypothetical acetamidase (AT10838). This cluster of ORFs is present in several distantly related strains including some beta and gamma proteobacteria, thus suggesting a horizontal gene transfer event. At present, nothing is known about the physiological role of this pathway, but its wide diffusion suggests a potentially important ecological role. The data related to Additional file 1: Figures S9-S11 are reported under "Additional file 2: Supplementary Results and Discussion".

\section{Stress response genes and their functions}

The PP1Y genome contains several ORFs potentially coding for the so-called resistance-nodulation-cell division (RND)-type efflux pumps [38] that actively excrete toxic molecules, and have thus been implicated in the capacity of PP1Y to grow in close contact with a diesel oil phase. (RND)-type efflux pumps are constituted by three subunits: the inner membrane, the outer membrane and the membrane fusion component. The PP1Y genome contains eight potential ORFs for the inner membrane subunit and even more for the other components (Additional file 1: Table S2), suggesting the possible formation of hybrid pumps. The evolutionary relationships among the inner membrane subunits are shown in Additional File 1: Figure S12A.

Three PP1Y RND pumps belong to a subfamily of pumps specific for neutral molecules like aromatic hydrocarbons, acriflavine and other toxic aromatic molecules. The product of AT9347 is closely related to toluene resistance proteins and is very likely an aromatic hydrocarbon resistance protein. Three PP1Y RND pumps belong to a subfamily specific for mono and divalent transition metals and are closely related to a set of RNDs pumps from Cupriavidus metallidurans $\mathrm{CH} 34$, a benchmark among strains able to tolerate very high concentrations of transition metals [39]. The PP1Y genome also contains eight potential ORFs for P-type ATPases (Additional file 1: Figure S12B), which are membrane ATP-dependent efflux pumps specialized in the excretion of metal cations [40]. For comparison, $C$. metallidurans $\mathrm{CH} 34$ genome codes for 9 P-type ATPases.

On the basis of these findings, we assayed the ability of PP1Y to grow in liquid medium containing high concentrations of metal cations. Figure 4 shows that PP1Y can grow in the presence of millimolar concentrations of nickel $(2.5 \mathrm{mM})$, lead $(10 \mathrm{mM})$, copper $(10 \mathrm{mM})$ and zinc $(5 \mathrm{mM})$. At higher concentrations, the growth rate steeply decreases to zero (not shown). Interestingly, all the metals increase the carbohydrates/proteins ratio with respect to the control culture, thus suggesting that modification of the cell envelope could contribute to resistance to metals. These results show that the ability of PP1Y to tolerate heavy metals is comparable to that of heavy metal-tolerating strains like C. metallidurans $\mathrm{CH} 34$ [40], which suggests that PP1Y could play a role in the bioremediation of hydrocarbons in environments polluted by heavy metals.

Tellurite anion is highly toxic to microorganisms (much more than arsenate and arsenite) thanks to its ability to catalyze the oxidation of cell thiols and produce radical oxygen species [41]. Therefore, the wide diffusion of tellurite-resistance mechanisms among bacteria is not surprising, and they might include an aspecific increase of the radical scavenger systems and specific tellurite anion transporters [42]. The PP1Y genome contains three ORFs potentially coding for proteins belonging to three different tellurite-resistance mechanisms: 


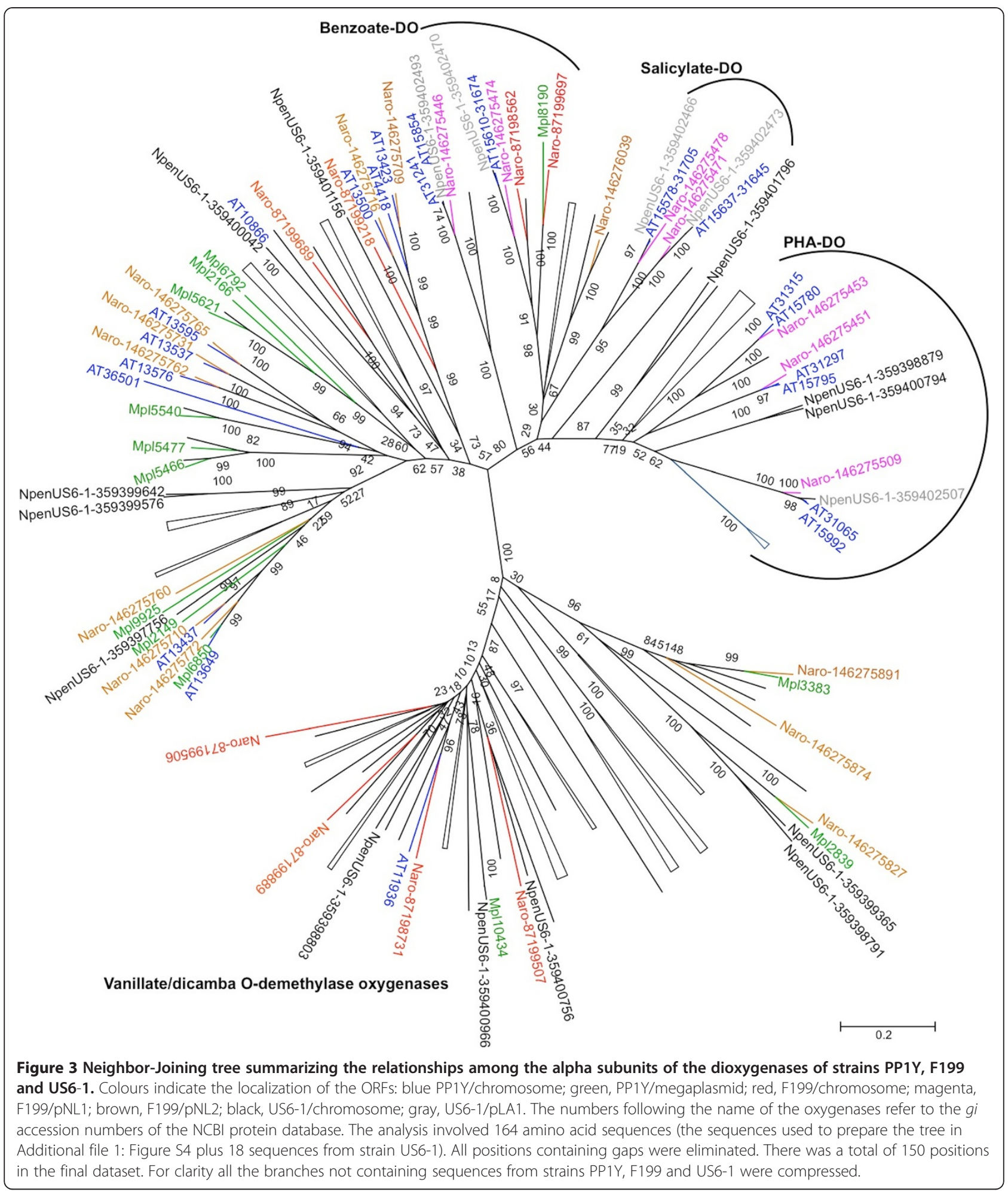

telA (from the E. coli kilA/telA/telB system), tehB (from the E. coli tehA/tehB system) and terC from Proteus mirabilis [43]. Due to the scarce knowledge about these systems, it is difficult to predict their role in tellurite resistance. However, all these ORFs are located in a cluster of ORFs coding for proteins probably involved in detoxification. Interestingly, a similar cluster of ORFs is present in the genome of strain RW1, but not in other sphingomonads (data not shown).

The importance of glutathione as a radical scavenger and mediator of detoxification systems varies among 
Table 5 PP1Y RCD specific activity assayed on 3-methylcatechol (3-MC), 2,3-dihydroxybiphenyl (2,3-DHBP), and 4-hydroxy-oestradiol (4-OHE)

\begin{tabular}{lcccc}
\hline Substrate & \multicolumn{4}{c}{ Enzyme specific activity (U/mg protein) } \\
\cline { 2 - 5 } & AT15599/AT31688 & Mpl3065 & AT32663 & AT15671/AT31616 \\
\hline 3-MC & 13.54 & 1.72 & 0.453 & 372.9 \\
2,3-DHBP & 32.73 & 12.05 & 23.5 & 48 \\
4-OHE & 10.33 & 0 & 44.62 & $3 \times 10^{-3}$ \\
\hline
\end{tabular}

AT numbers refer to the chromosome DNA.

bacteria. However, several bacteria use glutathione and glutathione-dependent enzymes to detoxify reactive organic compounds (like epoxides), halogenated compounds or alkylhydroperoxides, and reactive oxygen species (ROS) such as oxygen radicals [44].

In addition to genes involved in glutathione synthesis and in the reduction of oxidized glutathione, the PP1Y genome codes for 18 glutathione S-transferases (Additional file 1: Table S3). This number is about double that of E. coli and suggests that glutathione could play an important role in detoxification of toxic diesel oil components and of toxic metabolites produced by the oxidation of aromatic hydrocarbons, like epoxides and ROS.

The PP1Y genome also codes for six members of a peculiar family of very small (about 100 amino acids) monooxygenases known as "antibiotic biosynthesis monooxygenases" [45]. These enzymes are the only known monooxygenases not containing any metal or flavin cofactors [46], and that prevalently oxidize phenolic groups to quinines. They are involved in at least two very different physiological processes: (i) the synthesis of the polyketide antibiotics (e.g. the products of ActVA-Orf6 of
Streptomyces coelicolor), and (ii) the quinol redox cycle (e.g. quinol monooxygenase YgiN from E. coli). In particular, E. coli YgiN could prevent the accumulation of the semiquinone intermediate formed during the oxidation of quinols to quinones thus minimizing the formation of free radical species [47]. At least some of the six PP1Y antibiotic biosynthesis monooxygenases could have similar functions. However, some of them could be also involved in the synthesis of secondary metabolites. It is noteworthy that PP1Y is able to inhibit the growth of molds (unpublished results), which suggests it secretes antifungal compounds.

\section{Identification of genes involved in extracellular polymer secretion and biofilm formation}

The analysis of the PP1Y genome has revealed potential regulatory mechanisms (quorum sensing, QS) and secretion systems for extracellular polymers, including polysaccharides and poly-gamma-glutamate, which may play a role in the complex "social" behavior of PP1Y, a strain able to form different types of multicellular amorphous aggregates and ordered biofilm
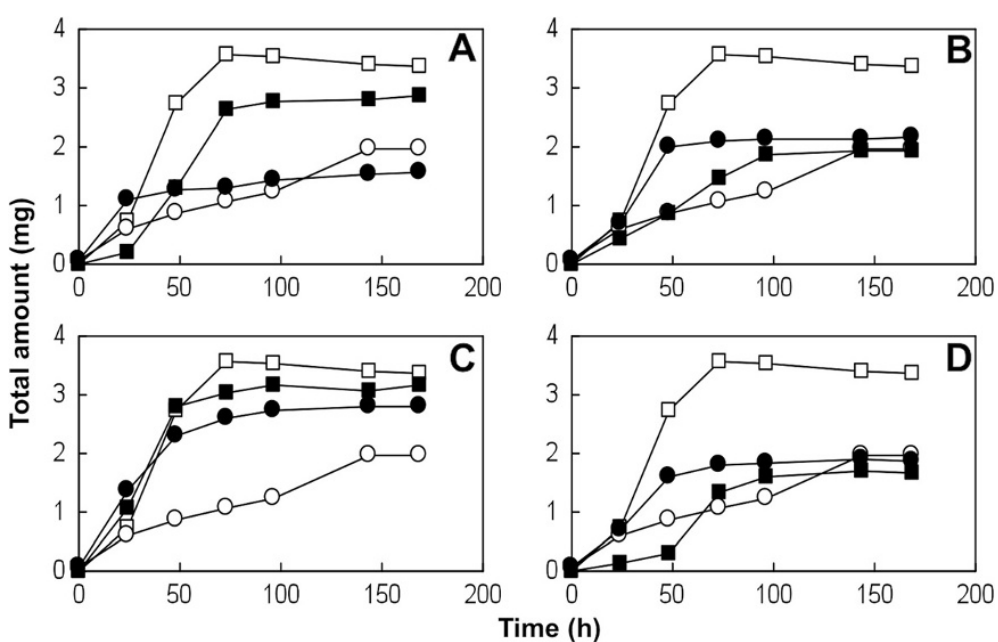

Figure 4 PP1Y growth, measured as total protein and total carbohydrate content in the culture medium, in the presence of millimolar concentrations of heavy metals. (A) $2.5 \mathrm{mM} \mathrm{NiCl}_{2 ;}$ (B) $10 \mathrm{mM} \mathrm{PbCl}$; (C) $10 \mathrm{mM} \mathrm{CuCl}_{2 ;}$ (D) $5 \mathrm{mM} \mathrm{ZnCl}_{2}$. The control growth shown in all graphs was performed in 1\% glutamic acid. Empty squares and circles: total proteins and total carbohydrates, respectively in the control culture. Filled squares and circles: total proteins and total carbohydrates, respectively in the cultures containing metals. Error bars are omitted for clarity; relative error was invariably lower than $8 \%$. 
(see also Additional file 2: Supplementary Results and Discussion). Quorum sensing is a simple molecular mechanism that results in coordinated behavior in response to cell density [48]. The presence in PP1Y of two QS systems is interesting since they could work simultaneously in response to two different cell densities or, could be activated alternatively under specific conditions. Both possibilities could account for PP1Y's complex behavior.

Although several ORFs for sphingan synthesis have distantly related homologues in the PP1Y genome (identity $<30-40 \%$ ), a gene cluster similar to those present in other Sphingomonas does not exist in PP1Y. Therefore, it is unlikely that PP1Y could produce a sphingan-like polysaccharide. However, several clusters potentially coding for the synthesis of extracellular polysaccharides are distributed among the larger replicons (chromosome and $\mathrm{Mpl}$ ), as shown in Additional file 1: Table S4, Table S5 and Figure S14A. Lpl contains two regions that are probably involved in the synthesis of exopolysaccharides (Additional file 1: Figure S14B), and are widely distributed among sphingomonads. The closest sequences can be found in S. japonicum UT26 with an identity of 70-90\% at protein level. Interestingly, the region between these two couples of ORFs in Lpl contains five ORFs coding for hypothetical glycosyl transferases and four ORFs coding for the subunits of an ABC-type polysaccharide transport system with high homology in several sphingomonads (Additional file 1: Figure S15 A-B). Lpl651 is particularly interesting as it codes for a large protein containing three glycosyl transferase-like domains. No other sphingomonad contains a representative of this subfamily of glycosyl transferases that can be found in distantly related bacteria, suggesting another case of horizontal gene transfer. Taken together these findings suggest that Lpl codes for the synthesis and export of one or more capsular polysaccharide(s) that probably contains mannose and rhamnose, like sphingans, but whose structures could differ from those produced by other sphingomonads.

Several biofilm-forming strains secrete cellulose as a matrix component. Lpl from PP1Y shares with Sphingobium japonicum UT26 a cluster of ORFs coding for a two-subunit cellulose synthase (Additional file 1: Figure S16A), which implicates Lpl in both biofilm synthesis and remodelling. Another CDS coding for a hypothetical cellulase is located on chromosome (AT36325) not far from a CDS coding for an exo-1,3/1,4-beta-glucanase which could act downstream the cellulase (endo-1,4-beta-glucanase) (Additional file 1: Figure S16B). Interestingly, PP1Y has the largest number of glycosyl hydrolases and glycosyl transferases among sphingomonadales and related groups of alpha proteobacteria (Additional file 1: Table S6).

The PP1Y genome contains three ORFs coding for $\gamma$-PGA polymerases (Additional file 1: Figure S16C), which are involved in the synthesis of poly-gammaglutamate, a strongly anionic homopolymer composed of glutamate residues linked by amide bonds between $\alpha$-amino and $\gamma$-carboxyl groups [49]. This polymer can perform different functions, including the stabilization of the extracellular matrix, glutamate storage and toxic metals binding (Additional file 2: Supplementary Results and Discussion).

\section{Conclusions}

This analysis of the annotated Novosphingobium sp. PP1Y genome has revealed peculiar biochemical and biotechnological properties, namely, the metabolic pathways specifically involved in: (i) the degradation of a vocabulary of aromatic hydrocarbons, (ii) the resistance to toxic compounds and (iii) the QS social behavior mechanism. This detailed functional evaluation opens new translational perspectives regarding the possible manipulation of the PP1Y genome for bioremediation purposes. Moreover, the comparison between the enzymatic machinery of PP1Y and those of the other sphingomonads able to degrade environmental pollutants suggests that each sphingomonad has independently evolved its own repertoire of degradative enzymes through a complex combination of vertical heredity, horizontal gene transfers, duplications and rearrangements. This process is still ongoing as demonstrated by the presence of multiple copies of pNL1-like regions at different locations of the PP1Y chromosome. As a consequence, even closely related strains like PP1Y, F199 and US6-1, which belong to the genus Novosphingobium, have unique features and adaptations to specific, also polluted, environments. The analysis reported in this paper strongly supports the general belief that sphingomonads are very adaptable bacteria with extraordinary genomic plasticity. It also raises biotechnological perspectives of using sphinomonads in bioremediation processes.

\section{Methods}

Bacterial growth and DNA extraction

Novosphingobium sp. strain PP1Y was routinely grown and genomic DNA was extracted as previously described [7].

\section{Genome sequencing and assembly}

The de novo whole-genome shotgun sequencing of Novosphingobium sp. PP1Y was carried out as described in a preliminary report (EMBL database under accession numbers: FR 856862, FR 856861, FR 856860 and FR 856859 for Chr, Mpl, Lpl and Spl, respectively) [20].

\section{Sequence annotation}

Sequence annotation includes predicted ORFs, rRNAs, tRNAs and other ncRNAs, identified by using the following tools: 
- ORFs were predicted by Grc [20] combined with the Uniref50 [21] and KEGG GENES [22] databases;

- rRNA genes and tRNAs, genes were identified by using RNAmmer [23] and tRNAScan-SE [24] respectively;

- Other predicted ncRNA elements were found by Infernal using the RFAM database records as models $[25,26]$.

In-house developed pipelines guided the whole annotation process, scheduling and running single applications on a 56-blade cluster. ORFs on chromosome, mega-, largeand small plasmids are identified by a number preceded by "AT", "Mpl", "Lpl" and "Spl" respectively. All the PP1Y ORFs and their protein sequences discussed in the text and/or included in the trees are available on the "Gene" database at http://www.ncbi.nlm.nih.gov/gene/.

\section{Phylogenetic analysis}

The sequences included in this study were selected by searching public protein databases with BLAST and PSI-BLAST [50]. Clustal Omega [http://www.ebi.ac.uk/ Tools/msa/clustalo/] was used to obtain multiple alignments. Alignments were visualized and examined using JalView [51] and MEGA5.1 [52]. Phylogenetic trees were obtained, visualized and manipulated using MEGA5.1. Bootstrap confidence analysis was performed on 1,000 replicates using the Neighbor-Joining method [53]. The evolutionary distances were computed using the Poisson correction method [54] and were expressed as the number of amino acid substitutions per site. All positions containing gaps and missing data were eliminated.

\section{Subcloning, expression and activity analysis of RCDs}

Open reading frames coding for RCDs were amplified by PCR using genomic DNA as template. Gene sequences were engineered to introduce an NdeI site at the 5'-end and a HindIII site at the 3 '-end. PCRs were performed in a total reaction volume of $50 \mu \mathrm{l}$, containing $50 \mathrm{ng}$ of genomic DNA, $1 \mu \mathrm{M}$ of each primer, $0.2 \mathrm{mM}$ dNTPs (Roche, Basel, Switzerland), $1 \times$ PCR buffer and $2.5 \mathrm{U}$ of Platinum pfx polymerase from Pyrococcus sp. (Invitrogen). The amplification program was optimized as follows: initial denaturation at $95^{\circ} \mathrm{C}$ for $2 \mathrm{~min}$, amplification for 20 cycles of denaturation at $92^{\circ} \mathrm{C}$ for $1 \mathrm{~min}$, annealing at $56^{\circ} \mathrm{C}$ for $1 \mathrm{~min}$, extension at $68^{\circ} \mathrm{C}$ for $1 \mathrm{~min}$. The amplified fragments cut with NdeI and HindIII were cloned into pET22b $(+)$ expression vector (Novagen) previously cut with the same enzymes. RCDs were expressed in E. coli strain BL21(DE3), transformed with the appropriate expression vector, purified by ionexchange chromatography on Q-Sepharose FF resin and analyzed for quality as described previously [55]. Assays were performed at $25^{\circ} \mathrm{C}$ in $50 \mathrm{mM} \mathrm{Tris} / \mathrm{HCl}(\mathrm{pH} 7.5)$ in a final volume of $500 \mu \mathrm{l}$ by spectrophotometric determination of the product of the reaction as described elsewhere [55]. The amount of the products was measured using their extinction coefficients: $\varepsilon_{388}=13,800 \mathrm{M}^{-1} \mathrm{~cm}^{-1}$ for the product of 3-methylcatechol (3-MC) [55]; $\varepsilon_{434}=$ $13,200 \mathrm{M}^{-1} \mathrm{~cm}^{-1}$ for the product of 2,3-dihydroxybiphenyl (2,3-DHBP) [56]; $\varepsilon_{298}=9,100 \mathrm{M}^{-1} \mathrm{~cm}^{-1}$ for the product of 4-hydroxy-oestradiol (4-OHE). One unit of enzyme activity was defined as the amount of enzyme required to form $1 \mu \mathrm{mol}$ of the product per minute under the assay conditions. Specific activity is given as units per milligram of protein.

Synthesis of 4-OHE was achieved by Dr. Pezzella (Department of Chemistry, University of Naples Federico II) via the o-Iodoxybenzoic acid (IBX)-mediated phenolic oxygenation procedure as previously described [57]. All chemicals were of the highest grade available and were from Amersham Biosciences, Promega, New England Biolabs, Sigma, ABCR GmbH, Fluka, or Applichem. Escherichia coli strain BL21 (DE3) and plasmid pET22b (+) were purchased from Novagen (Madison, WI, USA). DNA sequences and oligonucleotide synthesis were performed by Eurofins MWG Operon (Germany).

\section{Heavy metal resistance}

The resistance of bacteria to heavy metals was evaluated by measuring bacterial growth according to Notomista et al. [7] in a minimal medium containing $20 \mathrm{mM}$ MOPS pH 6.9, $100 \mathrm{mM} \mathrm{NaCl}, 1 \mathrm{~g} / \mathrm{L} \mathrm{NH}_{4} \mathrm{Cl}$ and $1.0 \%$ glutamic acid as sole carbon and energy source, plus trace amounts of four heavy metal salts: $\mathrm{NiCl}_{2}, \mathrm{CuCl}_{2}$, $\mathrm{ZnCl}_{2}$, and $\mathrm{PbCl}_{2}$, plus four heavy metal salts: $\mathrm{NiCl}_{2}$ (2.5 mM), $\mathrm{CuCl}_{2}(10 \mathrm{mM}), \mathrm{ZnCl}_{2}(5 \mathrm{mM})$, and $\mathrm{PbCl}_{2}$ (10 mM) (Sigma-Aldrich, St Louis, MO, USA).

\section{Availability of supporting data}

The following additional data are available with the online version of this paper: Additional file 2, which includes Supplementary Results and Discussion; and Additional file 1, which includes Tables S1 to S6 and Figures S1 to S16. Phylogenetic tree newick files are available online as Additional file 3. PP1Y genomic sequences are available in the EMBL database (http:// www.ebi.ac.uk/ena/) under accession numbers: FR 856862, FR 856861, FR 856860 and FR 856859 for Chr, $\mathrm{Mpl}$, Lpl and Spl, respectively (http://www.ebi.ac.uk/ena/ data/view/Taxon:Novosphingobium\%20sp.\%20PP1Y).

\section{Additional files}

Additional file 1: Supplementary Tables and Figures.

Additional file 2: Supplementary Tables and Figures.

Additional file 3: Phylogenetic tree newick files. 


\section{Abbreviations}

PAHs: Polycyclic aromatic hydrocarbons; Chr: Chromosome; Mpl: Mega plasmid; Lpl: Large plasmid; Spl: Small plasmid; ORFs: Open reading frames.

\section{Competing interests}

The authors declare that they have no competing interests.

\section{Authors' contributions}

VD, PC, BN and FS designed and performed the sequencing experiments; MP, LC and GP carried out the genome assembly and annotation; EN, VC, VI $L D, L T$ and ADD characterized and functionally evaluated PP1Y metabolic features; VD, EN, GP, FS and ADD analyzed the final data and wrote the manuscript. All the authors read and approved the final manuscript.

\section{Acknowledgment}

This work was supported by grant L.5/95 (to F.S.) from Regione Campania grant PS 35-126/Ind and grant PON01_02589 (MICROMAP) 2012 from the Ministry of University and Research (both to F.S.); grant RF-2010-2318372 from the Ministry of Health (to F.S.). The authors thank Jean Ann Gilder (Scientific Communication srl., Naples) for editing the text, and Vittorio Lucignano, CEINGE-Biotecnologie Avanzate for technical assistance.

\section{Author details}

${ }^{1}$ CEINGE-Biotecnologie Avanzate, Napoli, Italy. ${ }^{2}$ Dipartimento di Medicina Molecolare e Biotecnologie Mediche, Università di Napoli Federico II, Napoli, Italy. ${ }^{3}$ Dipartimento di Biologia, Università di Napoli Federico II, Napoli, Italy. ${ }^{4}$ Dipartimento di Medicina e Chirurgia, Università degli Studi di Salerno, Salerno, Italy. ${ }^{5}$ RCCCS-Fondazione SDN, Naples, Italy.

\section{Received: 5 November 2013 Accepted: 8 May 2014}

Published: 19 May 2014

\section{References}

1. Baird WM, Hooven LA, Mahadevan B: Carcinogenic polycyclic aromatic hydrocarbon-DNA adducts and mechanism of action. Environ $\mathrm{Mol}$ Mutagen 2005, 45:106-114.

2. Henry JA: Composition and toxicity of petroleum products and their additives. Hum Exp Toxicol 1998, 17:111-123.

3. King RW: Petroleum: its composition, analysis and processing. Occup Med 1988, 3:409-430

4. Wang Z, Fingas M, Blenkinsopp S, Sergy G, Landriault M, Sigouin L, Foght J, Semple K, Westlake DW: Comparison of oil composition changes due to biodegradation and physical weathering in different oils. J Chromatogr A 1998, 809:89-107.

5. Sawyer RF: Trends in auto emissions and gasoline composition. Environ Health Perspect 1993, 101:5-12.

6. Liang F, Lu M, Keener TC, Liu Z, Khang SJ: The organic composition of diesel particulate matter, diesel fuel and engine oil of a non-road diesel generator. J Environ Monit 2005, 7:983-988.

7. Notomista E, Pennacchio F, Cafaro V, Smaldone G, Izzo V, Troncone L, Varcamonti $M$, Di Donato A: The marine isolate Novosphingobium sp. PP1Y shows specific adaptation to use the aromatic fraction of fuels as the sole carbon and energy source. Microb Ecol 2011, 61:582-594.

8. Basta T, Keck A, Klein J, Stolz A: Detection and characterization of conjugative degradative plasmids in xenobiotic-degrading Sphingomonas strains. J Bacterio/ 2004, 186:3862-3872

9. Tabata M, Endo R, Ito M, Ohtsubo Y, Kumar A, Tsuda M, Nagata Y: The lin genes for $\gamma$-hexachlorocyclohexane degradation in Sphingomonas sp. MM-1 proved to be dispersed across multiple plasmids. Biosci Biotechnol Biochem 2011, 75:466-472.

10. Nagata $Y$, Natsui S, Endo R, Ohtsubo Y, Ichikawa N, Ankai A, Oguchi A, Fukui S, Fujita N, Tsuda M: Genomic organization and genomic structural rearrangements of Sphingobium japonicum UT26, an archetypal $\gamma$ hexachlorocyclohexane-degrading bacterium. Enzyme Microb Technol 2011, 49:499-508.

11. Copley SD, Rokicki J, Turner P, Daligault H, Nolan M, Land M: The whole genome sequence of Sphingobium chlorophenolicum L-1: insights into the evolution of the pentachlorophenol degradation pathway. Genome Biol Evol 2012, 4:184-198.

12. Fredrickson JK, Brockman FJ, Workman DJ, Li SW, Stevens TO: Isolation and characterization of a subsurface bacterium capable of growth on toluene, naphthalene, and other aromatic compounds. App/ Environ Microbiol 1991, 57:796-803.

13. Sohn JH, Kwon KK, Kang JH, Jung HB, Kim SJ: Novosphingobium pentaromativorans sp. nov., a high-molecular-mass polycyclic aromatic hydrocarbon-degrading bacterium isolated from estuarine sediment. Int J Syst Evol Microbiol 2004, 54:1483-1487.

14. Toyama T, Kainuma Y, Kikuchi S, Mori K: Biodegradation of bisphenol A and 4-alkylphenols by Novosphingobium sp. strain TYA-1 and its potential for treatment of polluted water. Water Sci Technol 2012, 66:2202-2208.

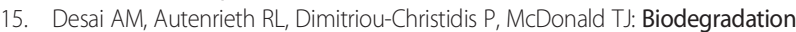
kinetics of select polycyclic aromatic hydrocarbon (PAH) mixtures by Sphingomonas paucimobilis EPA505. Biodegradation 2008, 19:223-233.

16. Coronado E, Roggo C, Johnson DR, van der Meer JR: Genome-wide analysis of salicylate and dibenzofuran metabolism in Sphingomonas wittichii RW1. Front Microbiol 2012, 3:300.

17. Kolvenbach BA, Corvini PF: The degradation of alkylphenols by Sphingomonas sp. strain TTNP3 - a review on seven years of research. Nat Biotechnol 2012, 30:88-95.

18. Romine MF, Stillwell LC, Wong KK, Thurston SJ, Sisk EC, Sensen C, Gaasterland T, Fredrickson JK, Saffer JD: Complete sequence of a 184-kilobase catabolic plasmid from Sphingomonas aromaticivorans F199. J Bacteriol 1999, 181:1585-1602

19. Miller TR, Delcher AL, Salzberg SL, Saunders E, Detter JC, Halden RU: Genome sequence of the dioxin-mineralizing bacterium Sphingomonas wittichii RW1. J Bacteriol 2010, 192:6101-6102.

20. D'Argenio V, Petrillo M, Cantiello P, Naso B, Cozzuto L, Notomista E, Paolella G, Di Donato A, Salvatore F: De novo sequencing and assembly of the whole genome of Novosphingobium sp. strain PP1Y. J Bacteriol 2011, 193:4296.

21. Warren AS, Setubal JC: The Genome Reverse Compiler: an explorative annotation tool. BMC Bioinforma 2009, 10:35.

22. Baris E, Suzek BE, Huang H, McGarvey P, Mazumder R, Wu CH: UniRef: comprehensive and non-redundant UniProt reference clusters. Bioinformatics 2007, 23:1282-1288.

23. Kanehisa M, Goto S: KEGG: kyoto encyclopedia of genes and genomes. Nucleic Acids Res 2000, 28:27-30.

24. Lagesen K, Hallin P, Rødland EA, Staerfeldt HH, Rognes T, Ussery DW: RNAmmer: consistent and rapid annotation of ribosomal RNA genes. Nucleic Acids Res 2007, 35:3100-3108.

25. Schattner $P$, Brooks AN, Lowe TM: The tRNAscan-SE, snoscan and snoGPS web servers for the detection of tRNAs and snoRNAs. Nucleic Acids Res 2005, 33:W686-W689.

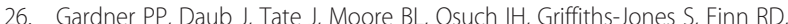
Nawrocki EP, Kolbe DL, Eddy SR, Bateman A: Rfam: Wikipedia, clans and the "decimal" release. Nucleic Acids Res 2011, 39:D141-D145.

27. Nawrocki EP, Kolbe DL, Eddy SR: Infernal 1.0: Inference of RNA alignments. Bioinformatics 2009, 25:1335-1337.

28. Gao F, Zhang CT: Ori-Finder: a web-based system for finding oriCs in unannotated bacterial genomes. BMC Bioinforma 2008, 9:79.

29. Gerdes K, Wagner EG: RNA antitoxins. Curr Opin Microbio/ 2007, 10:117-124.

30. Altschul SF, Gish W, Miller W, Myers EW, Lipman DJ: Basic local alignment search tool. J Mol Biol 1990, 215:403-410.

31. Nagata Y, Ohtsubo Y, Endo R, Ichikawa N, Ankai A, Oguchi A, Fukui S, Fujita $N$, Tsuda M: Complete genome sequence of the representative $\gamma$ hexachlorocyclohexane-degrading bacterium Sphingobium japonicum UT26. J Bacteriol 2010, 192:5852-5853.

32. Miyazaki $R$, Sato $Y$, Ito M, Ohtsubo $Y$, Nagata $Y$, Tsuda M: Complete nucleotide sequence of an exogenously isolated plasmid, pLB1, involved in gamma-hexachlorocyclohexane degradation. Appl Environ Microbiol 2006 72:6923-6933.

33. Moriya Y, Itoh M, Okuda S, Yoshizawa A, Kanehisa M: KAAS: an automatic genome annotation and pathway reconstruction server. Nucleic Acids Res 2007, 35:182-185.

34. Fuchs $\mathrm{G}$, Boll M, Heider J: Microbial degradation of aromatic compounds - from one strategy to four. Nat Rev Microbiol 2011, 9:803-816.

35. Cafaro V, Izzo V Scognamiglio R, Notomista E, Capasso P, Casbarra A, Pucci $P$, Di Donato A: Phenol hydroxylase and toluene/o-xylene monooxygenase from Pseudomonas stutzeri OX1: interplay between two enzymes. App/ Environ Microbiol 2004, 70:2211-2219.

36. Kim D, Chae JC, Zylstra GJ, Kim YS, Kim SK, Nam MH, Kim YM, Kim E: Identification of a Novel dioxygenase involved in metabolism of 
o-xylene, toluene, and ethylbenzene by Rhodococcus sp. Strain DK17. Appl Environ Microbiol 2004, 70:7086-7092.

37. Andújar E, Hernáez MJ, Kaschabek SR, Reineke W, Santero E: Identification of an extradiol dioxygenase involved in tetralin biodegradation: gene sequence analysis and purification and characterization of the gene product. J Bacteriol 2000, 182:789-795.

38. Paulson IT, Park JH, Choi PS, Saier MH: A family of Gram-negative bacterial outer membrane factors that function in the export of protein, carbohydrates, drugs, and heavy metals from Gram-negative bacteria. FEMS Microbiol Lett 1997, 156:1-8.

39. Janssen PJ, Van Houdt R, Moors H, Monsieurs P, Morin N, Michaux A, Benotmane MA, Leys N, Vallaeys T, Lapidus A, Monchy S, Médigue C, Taghavi S, McCorkle S, Dunn J, van der Lelie D, Mergeay M: The complete genome sequence of Cupriavidus metallidurans strain $\mathrm{CH} 34$, a master survivalist in harsh and anthropogenic environments. PLoS One 2010, 5:e10433.

40. Legatzki A, Grass G, Anton A, Rensing C, Nies DH: Interplay of the Czc system and two P-type ATPases in conferring metal resistance to Ralstonia metallidurans. J Bacteriol 2003, 185:4354-4361.

41. Chasteen TG, Fuentes DE, Tantaleán JC, Vásquez CC: Tellurite: history, oxidative stress, and molecular mechanisms of resistance. FEMS Microbiol Rev 2009, 33:820-832.

42. Joseph S, Desai P, Ji Y, Cummings CA, Shih R, Degoricija L, Rico A, Brzoska P, Hamby SE, Masood N, Hariri S, Sonbol H, Chuzhanova N, McClelland M, Furtado MR, Forsythe SJ: Comparative analysis of genome sequences covering the seven Cronobacter species. PLoS One 2012, 7:e49455.

43. Taylor DE, Rooker M, Keelan M, Ng LK, Martin I, Perna NT, Burland NT, Blattner FR: Genomic variability of $\mathrm{O}$ islands encoding tellurite resistance in Enterohemorrhagic Escherichia coli 0157:H7 isolates. J Bacteriol 2002, 184:4690-4698.

44. McLaggan D, Rufino H, Jaspars M, Booth IR: Glutathione-dependent conversion of $\mathrm{N}$-ethylmaleimide to the maleamic acid by Escherichia coli: an intracellular detoxification process. Appl Environ Microbiol 2000, 66:1393-1399

45. Valton J, Mathevon C, Fontecave M, Nivière V, Ballou DP: Mechanism and regulation of the two-component $\mathrm{FMN}$-dependent monooxygenase ActVA-ActVB from Streptomyces coelicolor. J Biol Chem 2008, 283:10287-10296.

46. Torres Pazmiño DE, Winkler M, Glieder A, Fraaije MW: Monooxygenases as biocatalysts: Classification, mechanistic aspects and biotechnological applications. J Biotechnol 2010, 146:9-24.

47. Bekker M, Alexeeva S, Laan W, Sawers G, Teixeira De Mattos J, Hellingwerf K: The ArcBA two-component system of Escherichia coli is regulated by the redox state of both the ubiquinone and the menaquinone pool. J Bacteriol 2010, 192:746-754

48. Li YH, Tian X: Quorum Sensing and Bacterial Social Interactions in Biofilms. Sensors 2012, 12:2519-2538.

49. Lemon KP, Earl AM, Vlamakis HC, Aguilar C, Kolter R: Biofilm development with an emphasis on Bacillus subtilis. Curr Top Microbiol Immunol 2008, 322:1-16.

50. Altschul SF, Madden TL, Schaffer AA, Zhang J, Zhang Z, Miller W, Lipman DJ: Gapped BLAST and PSI-BLAST: a new generation of protein database search programs. Nucleic Acids Res 1997, 25:3389-3402.

51. Waterhouse AM, Procter JB, Martin DM, Clamp M, Barton GJ: Jalview Version 2: a multiple sequence alignment editor and analysis workbench. Bioinformatics 2009, 25:1189-1191.

52. Tamura K, Peterson D, Peterson N, Stecher G, Nei M, Kumar S: MEGA5: molecular evolutionary genetics analysis using maximum likelihood, evolutionary distance, and maximum parsimony methods. Mol Biol Evol 2011, 28:2731-2739.

53. Saitou N, Nei M: The neighbor-joining method: A new method for reconstructing phylogenetic trees. Mol Biol Evol 1987, 4:406-425.

54. Zuckerkandl E, Pauling L: Evolutionary divergence and convergence in proteins. In Evolving Genes and Proteins. Edited by Bryson V, Vogel HJ. New York: Academic Press; 1965:97-166.

55. Viggiani A, Siani L, Notomista E, Birolo L, Pucci P, Di Donato A: The Role of the Conserved Residues His-246, His-199, and Tyr-255 in the Catalysis of Catechol 2,3-Dioxygenase from Pseudomonas stutzeri OX1. J Biol Chem 2004, 279:48630-48639.
56. Wesche J, Hammer E, Becher D, Burchhardt G, Schauer F: The bphC gene-encoded 2,3-dihydroxybiphenyl-1,2-dioxygenase is involved in complete degradation of dibenzofuran by the biphenyl-degrading bacterium Ralstonia sp. SBUG 290. J Appl Microbiol 2005, 98:635-645.

57. Pezzella A, Lista L, Napolitano A, D'Ischia M: An expedient one-pot entry to catecholestrogens and other catechol compounds via IBX-mediated phenolic oxygenation. Tetrahedron Lett 2005, 46:3541-3544.

doi:10.1186/1471-2164-15-384

Cite this article as: D'Argenio et al:: Complete sequencing of

Novosphingobium sp. PP1Y reveals a biotechnologically meaningful metabolic pattern. BMC Genomics 2014 15:384.

\section{Submit your next manuscript to BioMed Central and take full advantage of:}

- Convenient online submission

- Thorough peer review

- No space constraints or color figure charges

- Immediate publication on acceptance

- Inclusion in PubMed, CAS, Scopus and Google Scholar

- Research which is freely available for redistribution 